\title{
Overcoming of doxorubicin resistance in breast cancer cells by cationic peptides
}

\begin{abstract}
Background: Acquired resistance to various drugs is an obstacle to anticancer therapy. Doxorubicin (Dox) is the first-line chemo-drug for therapy of various malignant tumors but it fails when treatment of multi-drug resistant tumors. One of the main factors of Doxresistance is overexpression of drug resistance genes, particularly, P-glycoprotein - Pgp. Earlier we've revealed a high selective apoptosis of tumor cells, induced by some cationic peptides (CPs) in vitro through inactivation their cell targets - chaperone proteins nucleolin/ NCL and nucleophosmin/NPM. This paper describes effect of CPs on breast cancer (BC) cell lines HBL 100 and doxorubicin-resistant cell line HBL-100/Dox.
\end{abstract}

Objective: Is to examine the viability of $\mathrm{BC}$ and Dox-resistant $\mathrm{BC}$ cells after incubation with some CPs.

Results: Some Arg/Lys-enriched cationic peptides under study induce cell death by nucleolar stress mechanisms both in HBL 100 BC cell line and HBL 100/ID 120 one. So, this CPs is perspective agents for inducing apoptosis of $\mathrm{BC}$ cells and overcoming Dox resistance.

Keywords: breast cancer lines, doxorubicin resistance, nucleolin expression, cationic peptides, apoptosis induction
Volume 10 Issue 5 - 2019

\author{
Anna A Lushnikova,' Anastasia $\vee$ Onyan,' \\ Anna A Rudakova,' AlexandrV Kostarev, ${ }^{2}$ \\ Kseniya V Kozhikhova, ${ }^{3}$ Sergey M Andreev, ${ }^{3}$ \\ Natalya ' Moiseeva,' Mariya A Baryshnikova' \\ 'Carcinogenesis Institute, N.N. Blokhin Cancer Research Center, \\ Russia \\ ${ }^{2}$ Department of Physics, M.V. Lomonosov Moscow State \\ University, Russia \\ ${ }^{3} \mathrm{SSC}$ Immunology Institute, Russia
}

\begin{abstract}
Correspondence: Anna A Lushnikova, Carcinogenesis Institute, N.N. Blokhin Cancer Research Center, I I 5478 Kashirscoye Sh-24, Russia, Tel +74993235866 , Email LAN21@yandex.ru
\end{abstract}

Received: September 12, 2019 | Published: October 30, 2019
Abbreviations: CPs, cationic peptides; Dox, doxorubicin; $\mathrm{BC}$, breast cancer; ARE, AU-rich elements

\section{Introduction}

Drug resistance in tumor cells is one of the obstacles for effective cancer chemotherapy. Novel strategies for therapy of multi-drug resistant tumors include the combinations of doxorubicin with microRNA. ${ }^{1}$ Metformin $^{2}$ and other prodrugs. Various molecular constructions are used to codeliver doxorubicin +metformin followed by release metformin to enhance Dox cytotoxicity through attenuating hypoxic stress and inhibition of HIF $1 \alpha /$ Pgp expression. Small molecular inhibitors of oncoproteins and essential cell pathways are considered now as promising therapeutic agents.

Perspective tumor targets for these agents are nucleolar phosphoproteins with chaperone activities nucleolin, NCL and nucleophosmin, NPM. Both NCL and NPM are overexpressed in majority of tumors and subcellular (cell surface) NCL expression level is a marker of endothelial cells in angiogenic blood vessels. ${ }^{3}$ These proteins are involved in regulation of gene transcription and expression, including DNA replication and recombination, chromatin remodeling, RNA biogenesis and stability, molecular transport, signaling, etc. Cytosolic fractions of NCL and NPM binds with p53 and down regulate its activity. Moreover, NCL is involved in stabilization of BCL2 mRNA by binding of 3'UTR AU-rich elements (ARE) resulting in an increase of anti-apoptotic protein BCL2. Overexpressed NCL has been shown to mediate the antiangiogenic and antitumor activity of endostatin. ${ }^{4}$ Thus, both nucleolin and nucleophosmin play an important role in carcinogenesis. Cell surface nucleolin is characterized as a target for therapeutic intervention with antibody-related agents. ${ }^{5,6}$ Earlier we have found that some cationic peptides (CPs) are perspective as a ligands for NCL and NPM overexpressed in tumor cells. ${ }^{6}$ Activity of some CPs in relation to drug-resistant $\mathrm{BC}$ cells is firstly described and expected mechanisms are discussed.

\section{Materials and methods}

Breast cancer (BC) cell line HBL100 was purchased from Cytology Institute of Russian Academy of Sciences (S-Petersburg). The cells were cultured in DMEM medium (PanEco, Russia), in a humidified atmosphere in $5 \% \mathrm{CO}_{2}$ at $37^{\circ} \mathrm{C}$. Breast cancer doxorubicin-resistant cell line HBL-100/Dox was obtained by incubation of HBL-100 with doxorubicin $(0.3 \mathrm{mkM}) \mathrm{s}$ followed by selection resistant cell clones during 2 weeks. Cationic peptides (CPs) with different charge and molecular mass $\sim 2 \mathrm{kDa}$ were synthesized by solid phase method using the Fmoc-protective strategy. Cell viability was evaluated by standard MTT-assay using control (skin fibroblast line H1036) and BC cell lines HBL-100 and HBL-100/Dox, which were cultured 2-3 days in 96-well plates, $7 \times 10^{3}$ per well. The cells in complete growth media after $72 \mathrm{~h}$ were replaced with fresh media in absence or in presence of peptide water solution, $0,5-4 \mu \mathrm{g} / \mathrm{ml}$. After $72 \mathrm{~h}$, MTT aliquots of $20 \mu \mathrm{l}$ $(5 \mu \mathrm{g} / \mathrm{ml})$ were added per each well and plates were incubated at $37^{\circ} \mathrm{C}$ for $2-3 \mathrm{~h}$. The reaction was monitored at A490 $\mathrm{nm}$. Cell survival is reported as percentage of viable cells in the presence of the protein 
versus untreated cells. Typically, cell survival values were obtained from at least three independent experiments and three determinations were performed for each sample. Standard deviations were calculated for the results obtained from all the experiments.

Immunoblot analysis was used with proteins resolved from the cell lysates after SDS-PAGE followed by transfer to nitrocellulose membrane. The primary monoclonal antibodies against NCL, p53 and actin were obtained from Lab Biotech, Dia-m, Russia. Anti-actin rabbit polyclonal antibody (Dia-M, Abcam, Russia), HRP-conjugated anti-rabbit immunoglobulin's from goat antiserum, HRP-conjugated anti-mouse immunoglobulin's from goat antiserum (Dia-M/ Abcam, Russia) were also used. Pair molecular docking was performed using the program Maestro 11 to confirm the specific interaction between peptides and NCL/NPM. ${ }^{7,8}$

\section{Results}

Estimation of NCL and NPM expression in BC cell lines by immunohistochemical staining has revealed high levels both membrane and nucleolar fraction of NCL and nucleolar fractions of NPM versus normal cells. Differential expression levels of NCL and NPM in tumor and normal cells is related with proliferation rate and activation of cell signaling and metabolism in tumors. We firstly used synthetic cationic peptides for selective inhibition of BC and BC Doxresistant cell proliferation, revealed by fluorescent microscopy and MTT assay. Results of MTT assay, obtained on HBL- 100 and HBL100/Dox cell lines after $72 \mathrm{~h}$ cell cultivation and $2 \mathrm{~h}$ incubation with corresponding PC indicate, that viability of morphologically normal human fibroblasts (control line H1036) was $100 \pm 4.3 \%$ for these PC concentrations. However, significant decrease of $\mathrm{BC}$ cell viability induced by the tested PSs in concentrations of $0.5-4 \mu \mathrm{g} / \mathrm{ml}$ (Table 1). Breast cancer cells were sensitive to these peptide with $\mathrm{C}=1-4 \mathrm{mkg} / \mathrm{ml}$, since relative amounts of viable cells were lower $50 \%$, while normal skin fibroblasts continued the growth.

Table I Viability of HBL-I00 breast cancer cells and Dox-resistant HBL-I00/Dox cells after treatment with cationic peptides AM-2 and NC-783

\begin{tabular}{|c|c|c|c|c|}
\hline Cell line/CP dilution & $\mathrm{l}: 4$ ( 4mkg/ml) & I:8 ( 2mkg/ml) & I:16 ( / mkg/ml) & $0 \mathrm{I}: 32(0,5 \mathrm{mkg} / \mathrm{ml})$ \\
\hline \multicolumn{5}{|l|}{ HBL- 100} \\
\hline NC-783 & $5.4 \pm 0.5 \%$ & $7.5 \pm 0.9 \%$ & $40.1 \pm 3.2 \%$ & $72.8 \pm 6.5 \%$ \\
\hline AM-2 & $5.9 \pm 0.7 \%$ & $8.5 \pm 0.5 \%$ & $10.9 \pm 0.8 \%$ & $24.7 \pm 1.2 \%$ \\
\hline \multicolumn{5}{|l|}{ HBL 100/Dox } \\
\hline NC-783 & $25.4 \pm 1.6 \%$ & $35.7 \pm 1.8 \%$ & $43.0 \pm 2.8 \%$ & $78.2 \pm 6.9 \%$ \\
\hline AM-2 & $13.0 \pm 0.9 \%$ & $25.8 \pm 1.6 \%$ & $27.9 \pm 2.0 \%$ & $58.3 \pm 3.8 \%$ \\
\hline
\end{tabular}

Then we have analyzed a change of NCL, NPM, p53 and control actin levels before and after 24 h-incubation HBL line cells with cationic peptide. Western blotting indicates significant increase of p53 protein simultaneously with decrease the level of unbound NCL in a manner similar to levels of mRNA. These date confirm the apoptosis as a non-specific mechanism of $\mathrm{BC}$ and $\mathrm{BC} /$ Dox cell death induced by cationic peptides by nucleolar stress initiation.

The nucleolus functions as a compartment that is sensitive to cellular stress and isolates suppressor p53, which is released into nucleoplasm upon DNA damage. The mechanisms of protein sequestration into the nucleolus includes interaction of proteins with resident nucleolar proteins, namely NCL and NPM nucleolar localization signal (NoLS) enriched with $\mathrm{R} / \mathrm{K}$ amino acid residues. Cytotoxicity of cationic peptides under study is associated with ability to induce apoptosis. We have revealed that a number of Arg/Lys-rich cationic peptides are potential ligands for membrane NCL and form clusters, binding with active sites of NCL dimer and NPM followed by reducing its functional activity and degradation tumor cells by apoptosis and related processes Key amino acids that are significant for NCL-ligand binding seems to be Asp, Glu (q-, pH7.0 ), Arg, Lys(q+, pH7.0), Thr (q0, pH7.0), located in NCL and active center, including $\mathrm{RB}$ regions and forming hydrogen bounds with some $\mathrm{NH}_{2}$ groups in peptide molecule (Figure 1). The docking was performed by glide 6.0 in total, 4 configurations for each conformer and score function GS per the configuration were calculated. Interestingly, that majority of the peptide fragments had a high score $>6.00 \mathrm{kcal} / \mathrm{mol}$ (value modules). All these structures located in approximate active centre of the proteins NCL and NPM, and formed hydrogen bonds between the same amino acids. This fact indicates the characteristic regions of binding between peptides and their targets - NCL and NPM. All the cell lines under study have revealed a high selective cytotoxicity for tested CPs: IC50 ranged from 0.5 to $1 \mu \mathrm{g}$, with induction of apoptosis. Control fibroblast survival has not changed significantly. In the course of 2-6hours of cultivation of tumor cells in 96 well cell cultured clusters and micro-wells, activation of caspase 3, 8 and 9 (HBL-100) or caspase 9 (HBL-100/Dox) was detected along with increased frequency of DNA double-strand breaks, degradation of chromatin and mitosis spindle. Moreover, CPs inhibits Pgp expression and depletes ATP production, inducing mitochondrial toxicity. Tumor cell apoptosis was revealed in breast cancer line HBL-100/Dox, apparently associated with the activation of caspase 9, 2 and NCL-RFWD-p53 signal cascade. Molecular interactions were confirmed by pair docking. A modelling of the interactions of CPs with cell surface NCL and NPM has revealed a high scores and preferred amino acid residues that are forming hydrogen bonds with these proteins (Figure $2 \& 3$ ). 

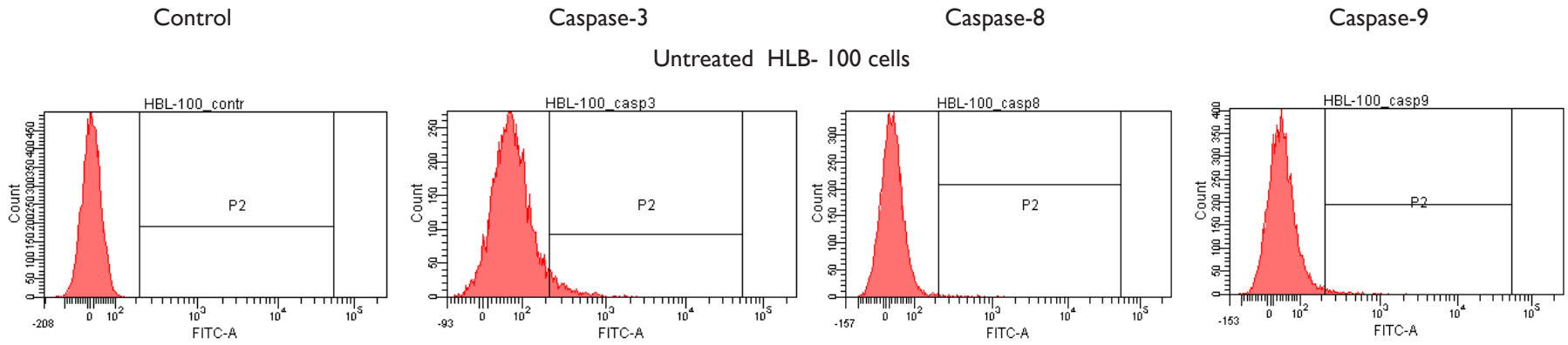

$5.1 \%$

$0.6 \%$

$1.2 \%$

HB- 100 cells, treated with CP AM2
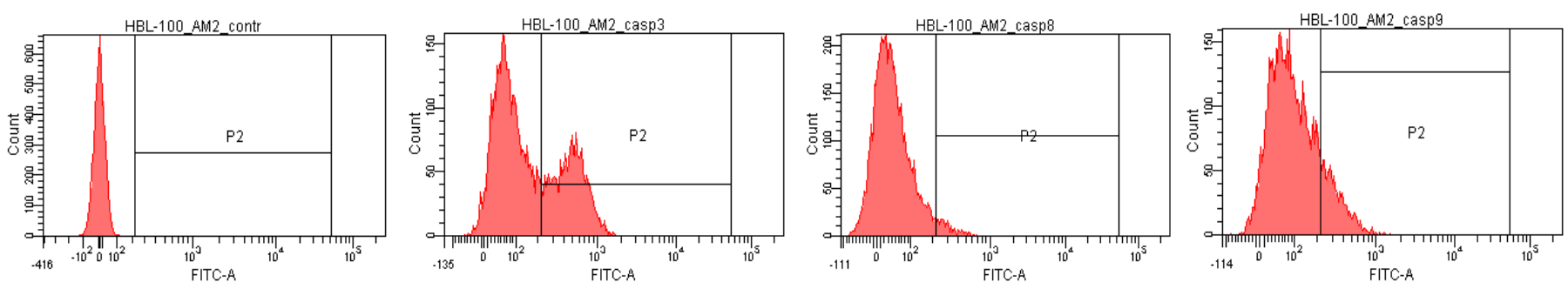

$34.8 \%$

$3.3 \%$

$15.2 \%$

Control

Caspase-3

Caspase-8

Caspase-9

Untreated HLB-100/Dox cells

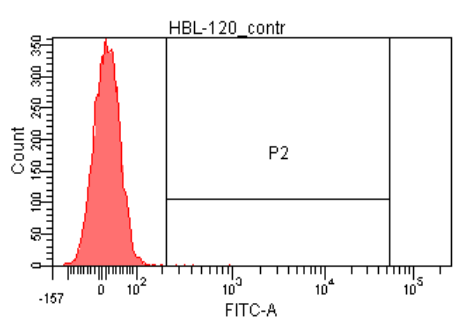

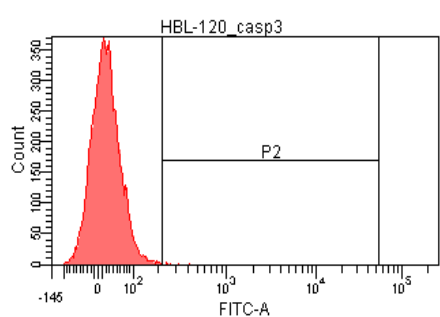

$0.1 \%$

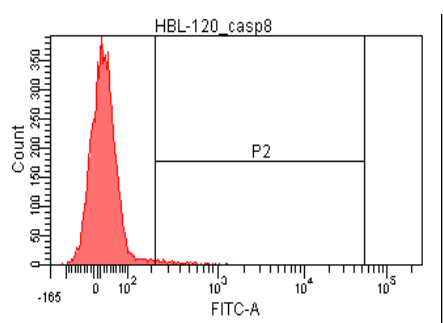

$1.6 \%$

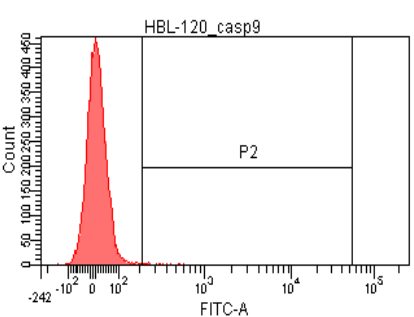

$0.3 \%$

HB- 100/Dox cells, treated with CP AM2

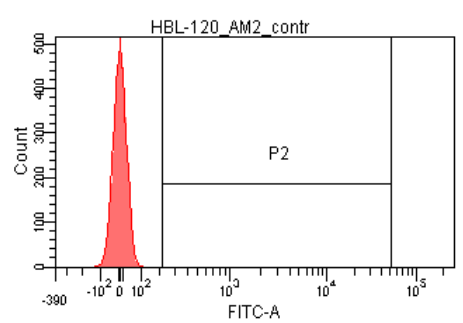

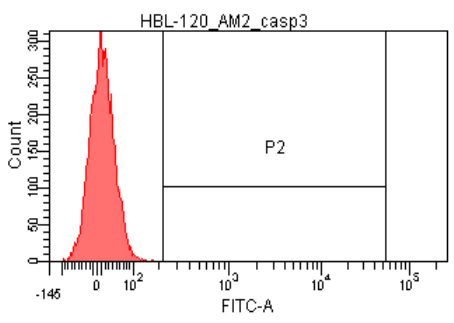

$0.1 \%$

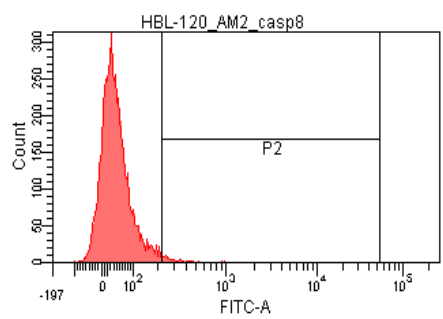

$1.5 \%$

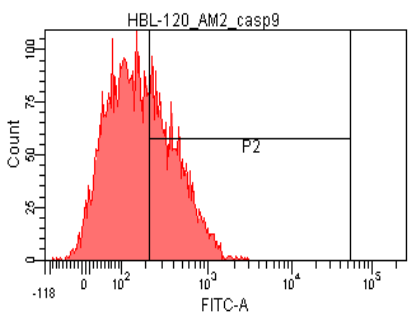

$37.5 \%$

Figure I Activation of caspase 3,8,9 and caspase 9 after incubation HBL 100 and HBLI00/Dox cells with cationic peptide AM-2. 

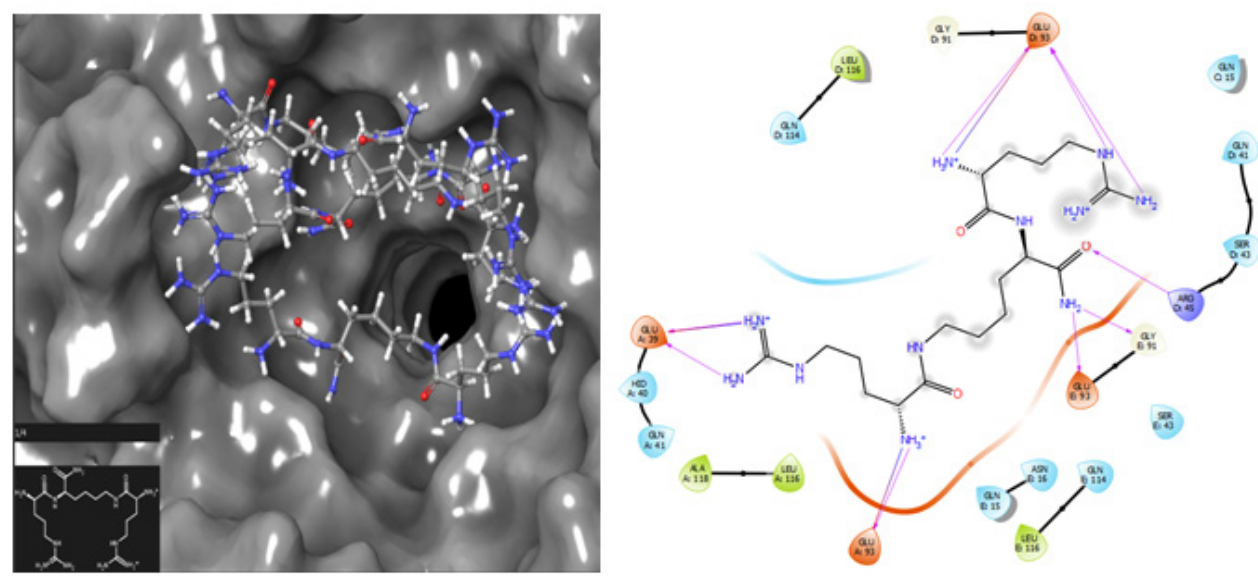

A

B

Figure 2 (A) Docking CP NC8II to NPM; (B) hydrogen bonds between CP and Glu 39,93,Arg45, Gly 9 I amino acids in NPM active center are shown.
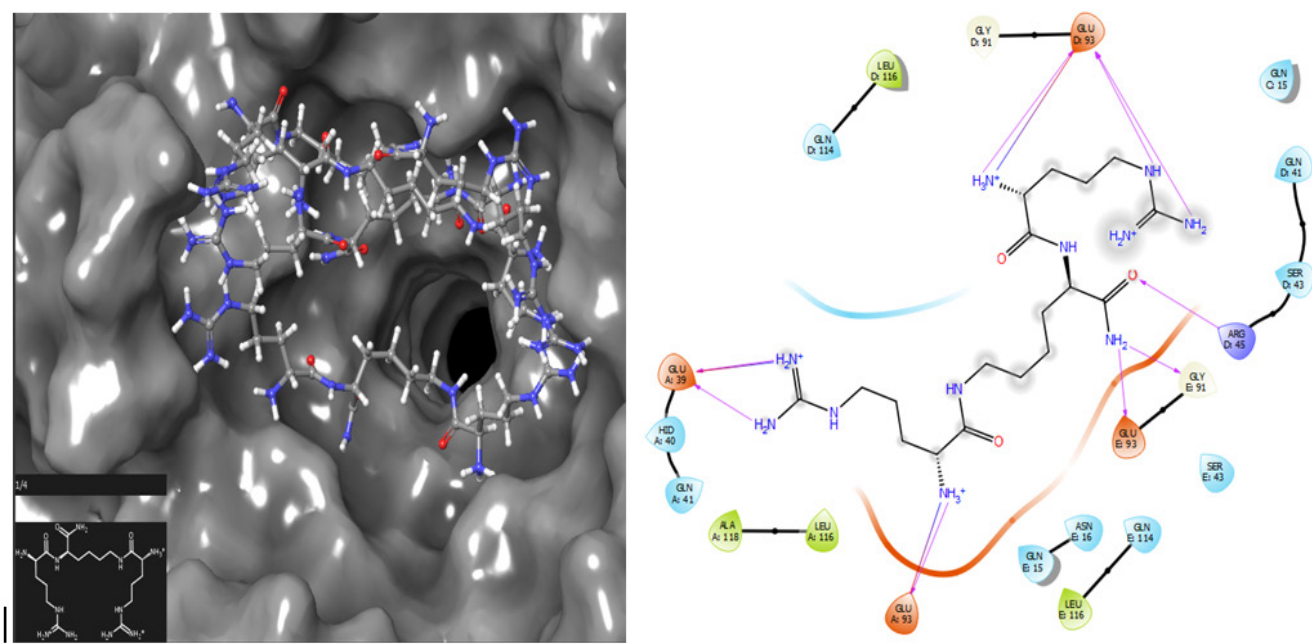

Figure 3 (A) Docking NC 783 to NCl; (B) hydrogen bonds between CP and Asp 60, 87, Thr88 amino acids in NCL active center are shown.

\section{Conclusion}

Some Arg/Lys-enriched cationic peptides study induces apoptosis by nucleolar stress mechanisms both in HBL $-100 \mathrm{BC}$ cell line and doxorubicin-resistant HBL- 100/Dox line. This CPs is considered as perspective agents for inducing apoptosis of $\mathrm{BC}$ cells and overcoming Dox resistance.

\section{Acknowledgments}

The authors are appreciating to Dr. Lidia F Morosova for useful discussions, advices and support.

\section{Conflicts of interest}

The authors declare that there no conflict of interest.

\section{References}

1. Rui M, Qu Y, Gao T, et al. Simultaneous delivery of anti-miR21 with doxorubicin prodrug by mimetic lipoprotein nanoparticles for synergistic effect against drug resistance in cancer cells. Int $J$ Nanomedicine. 2016;12:217-237.

2. Li Y, Luo J, Lin MT, et al. Co-delivery of metformin enhances the antimultidrug resistant tumor effect of doxorubicin by improving hypoxic tumor microenvironment. Mol Pharm. 2019;16(7):2966-2979.

3. Chrostian S, Pitch J, Akerman ME, et al. Nucleolin expression at the sell surfaces is a marker of endothelial cells in angiogenic blood vessels. $J$ Cell Biol. 2003;163(4):871-878.

4. Shi H, Huang $Y$, Zhou $H$, et al. Nucleolin is a receptor that mediates antiangiogenic and antitumor effect of endostatin. Blood. 2007;110(8):2899-2906.

5. Berger CM, Gaume X, Bouvet P. The roles of nucleolin subcellular localization in cancer. Biochimie. 2015;113:78-85.

6. Romano S, Fonseca N, Simões S, et al. Nucleolin-based targeting strategies for cancer therapy: from targeted drug delivery to cytotoxic ligands. Drug Discov Today. 2019. 
7. Lushnikova A, Ponkratova D, Andreev S, et al. A possibility for therapy of metastatic cutaneous melanoma with cationic peptides. Europ J Cancer. 2017;72 ( Suppl ):127-128
8. Lushnikova A, Kostarev AV, Onyan AV, et al. Simulation binding between nucleolin and cationic peptides, inducing tumor cell apoptosis, by molecular docking. J Cancer Prev Curr Res. 2018;9(4):187-189. 